\title{
Application of Dijkstra algorithm in open evaluation of community
}

\author{
Zengji Wang \\ School of North China Electric Power University, Baoding 071003, China; \\ 1379130580@qq.com
}

Keywords: Dijkstra algorithm; closed community; Brasee paradox;

\begin{abstract}
.
The Chinese government has formulated a policy that, in principle, is no longer a closed community. The existing closed area and unit compound should open gradually. The policy has aroused widespread concern and discussion. We use the Dijkstra algorithm to simulate the district road network. Based on the study of the total time before and after the opening of the District, this paper studies whether the district is open or not. This method has two advantages, quantitative answers to the impact of the open area on the road network, and can find the Brasee paradox. Finally, we have a case study of a district in Changsha, China.
\end{abstract}

\section{Introduction}

In addition to the open area may lead to security issues, the focus of the discussion is: open area can achieve the optimization of network structure, improve the road capacity, to improve traffic conditions, and how to improve the effect of.Some people think Gated Community destroy the structure of urban road network.It is easy to cause traffic jams. After the opening of the district, the road network density and road area increased, so traffic capacity will naturally improve. ${ }^{[1]}$ It is also considered that the area, location, external and internal road conditions and many other factors. ${ }^{\text {[2][3] }}$ The specific situation is very complex.

\section{Dijkstra algorithm $^{[4]}$}

We build a weighted graphs $G=(V, E, W)$. V means every intersection. $E$ is a set of edges, that is, the road connecting each intersection in the graph. Adjacency matrix $\mathrm{W}_{\mathrm{ij}}(\mathrm{n} \times \mathrm{n})$ means the time impedance between vi and vj. If there is no road between $v_{i}$ and $v_{j}, W_{i j}=\infty$. Dijkstra algorithm is a good method to find the shortest path.

\section{Simulation Process}

Due to the development of the network, we believe that each driver will choose the route that takes the least time. As a result of human egoism, making the choice of the least time-consuming path, we will not take into account the impact on others. So the final system is not the best time. Now, the paper gives a method of simulating the walking path of the road network.

The simulation is as follows: A driver start from A point to B point. Using the Dijkstra algorithm to select the shortest path, the driver starts. When the next driver chooses the route, due to the shortest time on the road increases, we need to calculate the time spent on the road again. Then the driver starts again. And so on, until the number of vehicles achieve the desired value. 


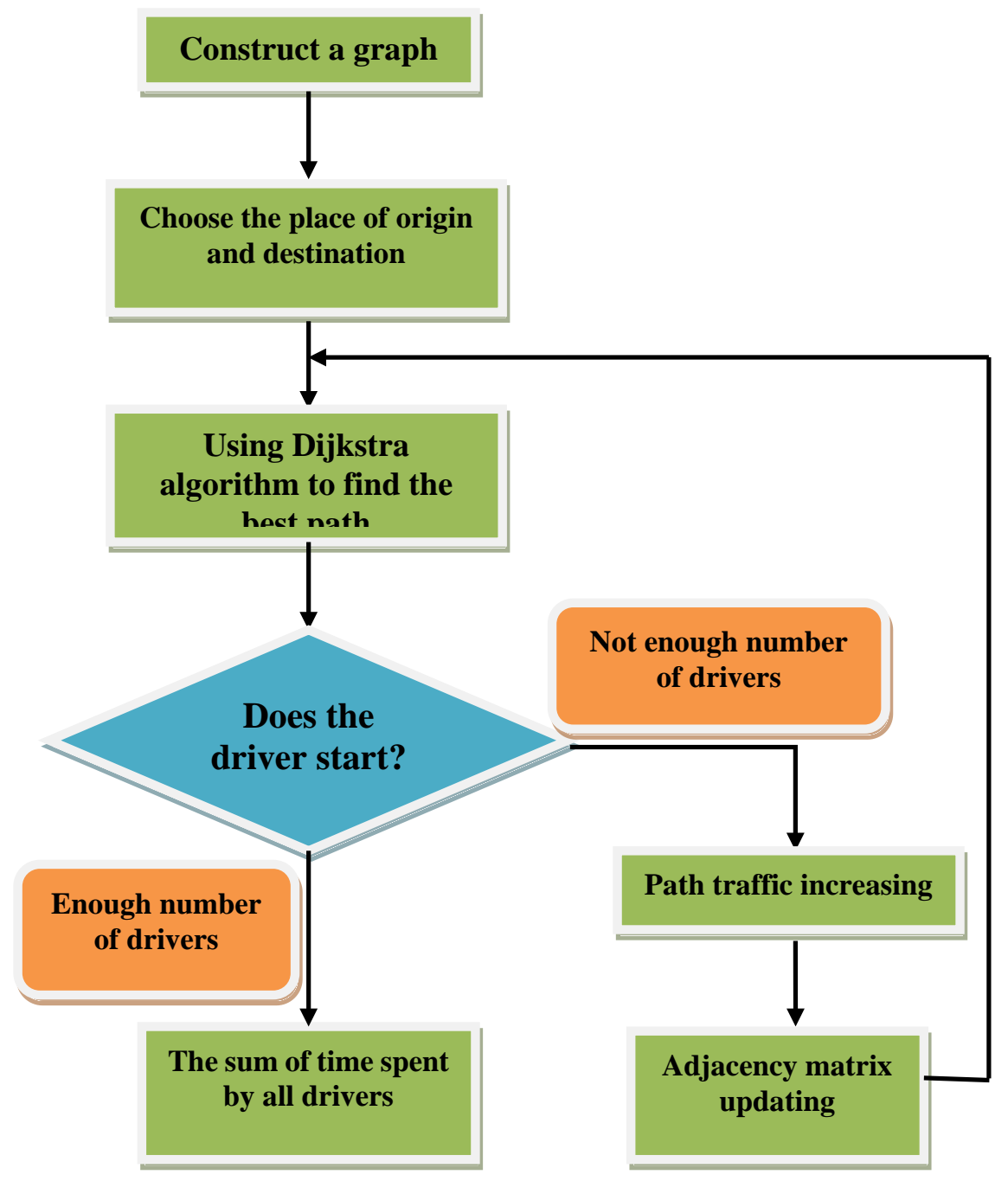

\section{Example calculation}

We study the Changsha a district. It was abstracted as below, which is part of the shadow area. Left figure is the closed one and right is after opening. The impedance between two points is described in the literature. ${ }^{[5]}$
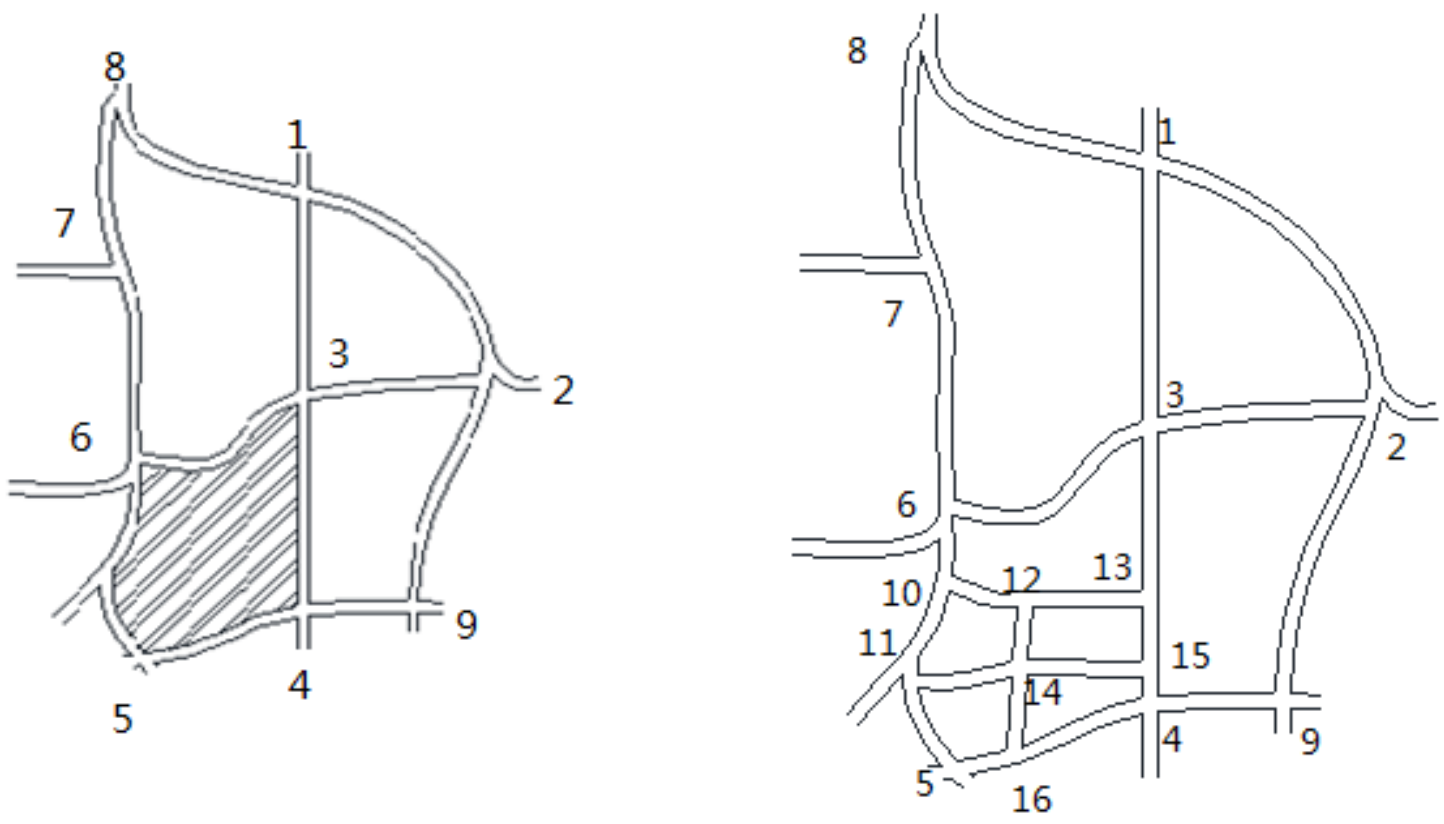
We choose from 4 to 6 , from 8 to 9 as two routes. And simulate to get the total time spent. And the result as follows:

From 8 to 9

\begin{tabular}{ccc}
\hline mode & closed & open \\
\hline Total time(second) & 6424856 & 7156434 \\
\hline From 4 to 6 & & \\
\hline mode & closed & open \\
\hline Total time(second) & 973567 & 1467065 \\
\hline
\end{tabular}

As can be seen, in this case, the district opened to make people travel less time. And the closer to the plot, the impact is greater. If the flow of these two routes accounted for the majority of people in this region, then I think this area should be open

\section{Verification of the Braess paradox ${ }^{[6]}$}
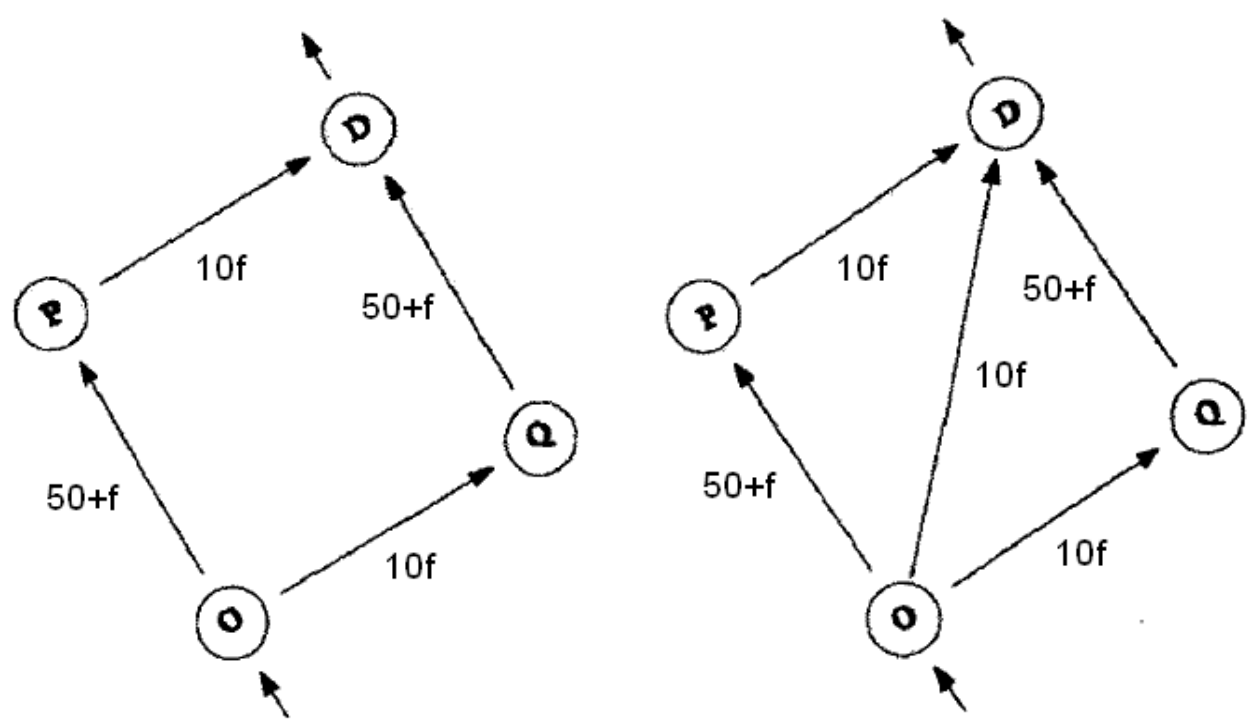

Study the above two road network. A person leaves from $O$ point to $D$ point. ' $f$ ' means the number of vehicle in the route. We assume that the total traffic flow from $\mathrm{O}$ to $\mathrm{D}$ is 6 . We can figure out that the total time required in Figure 1 is 498, and the total time required in Figure 2 is 552. This method reflects the Braess paradox.

\section{Conclusion}

Using the Dijkstra algorithm to simulate the open area, we can find the change of the surrounding road after opening. It can also find out the fact that the community is open but the total travel time increases. The method can be used to simulate the policy of open or not, and the results have reference value.

\section{References}

[1]B. Qiu. Why closed area should be opened. Xinhua Daily,2016-03-23(015). 
[2]L. Li, X. Wang. Open Gated Community: Game of advantage and disadvantage. Zhongzhou Construction, 2016(8):6-12.

[3]W.Liu. Study on complex network model and congestion of urban road traffic system. Zhengzhou University,2015.

[4] S. Wang, Z. Wu. Improved Dijkstra algorithm and its application [J]. Computer Science, 2012,(05):223-228

[5] X.Li. Countermeasures of urban traffic congestion: A Study on traffic opening of closed community. Changsha University of Science and Technology, 2014.

[6] W. Liu, Q. Zeng. Computer Engineering and Design, [J]. Braess paradox based on complex network 2015, (04): 1098-1102 